\title{
The structure of Sobolev extension operators
}

\author{
Charles Fefferman, Arie Israel and Garving K. Luli
}

\begin{abstract}
Let $L^{m, p}\left(\mathbb{R}^{n}\right)$ denote the Sobolev space of functions whose $m$-th derivatives lie in $L^{p}\left(\mathbb{R}^{n}\right)$, and assume that $p>n$. For $E \subseteq \mathbb{R}^{n}$, denote by $L^{m, p}(E)$ the space of restrictions to $E$ of functions $F \in L^{m, p}\left(\mathbb{R}^{n}\right)$. It is known that there exist bounded linear maps $T: L^{m, p}(E) \rightarrow L^{m, p}\left(\mathbb{R}^{n}\right)$ such that $T f=f$ on $E$ for any $f \in L^{m, p}(E)$. We show that $T$ cannot have a simple form called "bounded depth".
\end{abstract}

\section{Introduction}

Let $\mathbb{X}$ denote any of the following standard function spaces on $\mathbb{R}^{n}$ :

- $\mathbb{X}=C^{m}\left(\mathbb{R}^{n}\right)$, the space of real-valued $F \in C_{\text {loc }}^{m}\left(\mathbb{R}^{n}\right)$ for which the norm

$$
\|F\|_{C^{m}\left(\mathbb{R}^{n}\right)}:=\sup _{x \in \mathbb{R}^{n}} \max _{|\alpha| \leq m}\left|\partial^{\alpha} F(x)\right| \text { is finite; }
$$

- $\mathbb{X}=C^{m, s}\left(\mathbb{R}^{n}\right)$, the space of all functions $F \in C^{m}\left(\mathbb{R}^{n}\right)$ for which the norm

$$
\|F\|_{C^{m, s}\left(\mathbb{R}^{n}\right)}:=\|F\|_{C^{m}\left(\mathbb{R}^{n}\right)}+\sup _{\substack{x, y \in \mathbb{R}^{n} \\ x \neq y}} \max _{|\alpha|=m} \frac{\left|\partial^{\alpha} F(x)-\partial^{\alpha} F(y)\right|}{|x-y|^{s}}
$$

is finite (here $0<s<1$ );

- $\mathbb{X}=L^{m, p}\left(\mathbb{R}^{n}\right)$, the homogeneous Sobolev space of all real-valued functions $F$ for which the seminorm

$$
\|F\|_{L^{m, p}\left(\mathbb{R}^{n}\right)}:=\left\|\nabla^{m} F\right\|_{L^{p}\left(\mathbb{R}^{n}\right)} \text { is finite. }
$$

(Here, we take $p>n$, so that $\mathbb{X} \subseteq C_{\text {loc }}^{m-1,1-n / p}\left(\mathbb{R}^{n}\right)$, by the Sobolev theorem.)

For $E \subseteq \mathbb{R}^{n}$, we set $\mathbb{X}(E):=\left\{\left.F\right|_{E}: F \in \mathbb{X}\right\}$, equipped with the seminorm

$$
\|f\|_{\mathbb{X}(E)}:=\inf \left\{\|F\|_{\mathbb{X}}: F \in \mathbb{X}, F=f \text { on } E\right\} .
$$

Mathematics Subject Classification (2010): Primary 41A05; Secondary 46E35.

Keywords: Whitney extension problem, linear operators, Sobolev spaces. 
Let $A \geq 1$ be a real number. An extension operator for $\mathbb{X}(E)$ with norm $A$ is a linear map $T: \mathbb{X}(E) \rightarrow \mathbb{X}$ such that for all $f \in \mathbb{X}(E)$ we have

$$
T f=f \quad \text { on } E
$$

and

$$
\|T f\|_{\mathbb{X}} \leq A\|f\|_{\mathbb{X}(E)}
$$

For $\mathbb{X}=C^{m}\left(\mathbb{R}^{n}\right)$ or $C^{m, s}\left(\mathbb{R}^{n}\right)$ and $E \subseteq \mathbb{R}^{n}$ arbitrary, there exists an extension operator whose norm depends only on $m$ and $n$. Similarly, for $\mathbb{X}=L^{m, p}\left(\mathbb{R}^{n}\right)$ and $E$ arbitrary, there exists an extension operator whose norm depends only on $m, n$ and $p$. See [1], [2], and [4].

We want to know whether such extension operators can be taken to have a simple form when $E$ is finite. Recall that any linear map $T: \mathbb{X}(E) \rightarrow \mathbb{X}\left(E \subseteq \mathbb{R}^{n}\right.$ finite) has the form

$$
T f(x)=\sum_{y \in E} \lambda(x, y) f(y) \quad\left(\text { all } x \in \mathbb{R}^{n}\right),
$$

with coefficients $\lambda(x, y)$ independent of $f$. Let $D$ be a positive integer. We say that $T$ has depth $D$ if, for each fixed $x$, at most $D$ of the coefficients $\lambda(x, y)$ are nonzero.

Let $\mathbb{X}=C^{m}\left(\mathbb{R}^{n}\right)$ or $C^{m, s}\left(\mathbb{R}^{n}\right)$, and let $E \subseteq \mathbb{R}^{n}$ be finite. Then there exists an extension operator for $\mathbb{X}(E)$, whose norm and depth depend only on $m$ and $n$. See [1] and [3].

Thus, it is natural to ask the following:

Let $\mathbb{X}=L^{m, p}\left(\mathbb{R}^{n}\right)$, and let $E \subseteq \mathbb{R}^{n}$ be finite. Does there exist an extension operator for $\mathbb{X}(E)$, whose norm and depth depend only on $m, n$ and $p$ ?

Unfortunately, the answer is NO. In this paper, we establish the following result.

Theorem 1. Let $p>2, A \geq 1$ and $D \geq 1$ be given. Then there exists a finite set $E \subseteq \mathbb{R}^{2}$ such that $L^{2, p}(E)$ has no extension operator of norm $A$ and depth $D$.

More precisely, for $N \geq 2$, let

$$
E_{N}:=\left\{\left(2^{-k},\left(2^{-k}\right)^{2-2 / p}\right): k=2, \ldots, N\right\} \cup\{(0,0)\} \subseteq \mathbb{R}^{2} .
$$

Theorem 2. Let $p>2, A \geq 1, D \geq 1$, and let $0<\epsilon<3 / p$. If $L^{2, p}\left(E_{N}\right)$ has an extension operator with norm $A$ and depth $D$, then

$$
A \cdot D^{5 / p}>c(\epsilon, p) \cdot N^{\epsilon}, \quad \text { where } c(\epsilon, p) \text { depends only on } \epsilon \text { and } p \text {. }
$$

Theorem 2 will be proven in the next section. Theorem 1 follows at once from Theorem 2.

We mention a few related results in the literature. For $\mathbb{X}=C^{m, s}\left(\mathbb{R}^{n}\right)$, Luli [6] constructed extension operators of bounded depth without the assumption that $E$ is finite. The analogous result for $\mathbb{X}=C^{m}\left(\mathbb{R}^{n}\right)$ is false; however, there exist extension operators of "bounded breadth". (See [3].) For $\mathbb{X}=L^{m, p}\left(\mathbb{R}^{n}\right)$ and $E$ finite, an extension operator may be taken to have "assisted bounded depth"; see [4]. 
Acknowledgements. This work was developed during a workshop hosted by the American Institute of Mathematics (AIM). We would like to thank the NSF, ONR, AIM, and the workshop organizers for their generosity.

\section{Proof of Theorem 2}

Fix $p>2$ and $0<\epsilon<1 /(3 p)$, and let $\alpha:=1-2 / p$. Unless stated otherwise, $C, c$, etc. denote constants depending only on $p$, which may change value from one occurrence to the next.

For any $C^{1}$ function $F: \mathbb{R}^{2} \rightarrow \mathbb{R}$ and $y \in \mathbb{R}^{2}$, let $J_{y} F$ denote the first order Taylor polynomial of $F$ at $y$ :

$$
\left(J_{y} F\right)(x)=F(y)+\nabla F(y) \cdot(x-y) .
$$

We require $p>2$ so that the Sobolev theorem holds. In particular, after modification on some measure zero subset, each $F \in L^{2, p}\left(\mathbb{R}^{2}\right)$ belongs to $C_{\text {loc }}^{1, \alpha}\left(\mathbb{R}^{2}\right)$ and satisfies the inequalities:

$$
\begin{aligned}
|\nabla F(x)-\nabla F(y)| & \leq C\|F\|_{L^{2, p}\left(\mathbb{R}^{2}\right)}|x-y|^{\alpha} \\
\left|F(x)-J_{y} F(x)\right| & \leq C\|F\|_{L^{2, p}\left(\mathbb{R}^{2}\right)}|x-y|^{1+\alpha} \quad\left(\text { all } x, y \in \mathbb{R}^{2}\right) .
\end{aligned}
$$

We extend the $L^{2, p}$ norm to $\mathbb{R}^{2}$-valued functions by setting $\|\Psi\|_{L^{2, p}\left(\mathbb{R}^{2}\right)}:=\left\|\Psi_{1}\right\|_{L^{2, p}\left(\mathbb{R}^{2}\right)}+\left\|\Psi_{2}\right\|_{L^{2, p}\left(\mathbb{R}^{2}\right)}$, where $\Psi=\left(\Psi_{1}, \Psi_{2}\right)$ in coordinates.

We define the curve $\gamma:=\left\{\left(s, s^{1+\alpha}\right): s \in[0,1]\right\} \subseteq \mathbb{R}^{2}$. Let $N \geq 2$. We write $E$ for the subset $E_{N}$ defined in the introduction:

$$
E:=\left\{\left(2^{-k},\left(2^{-k}\right)^{1+\alpha}\right): k=2, \ldots, N\right\} \cup\{(0,0)\} \subseteq \gamma .
$$

In proving Theorem 2, it suffices to assume that $N$ is sufficiently large. More precisely, we henceforth assume that

(2.3) $N \geq Z$, where $Z \geq 1$ is some large constant that depends only on $p$ and $\epsilon$.

We determine $Z$ through Lemma 1 below.

Lemma 1. There exists $Z \geq 1$ depending only on $p$ and $\epsilon$, such that the following holds. Assume (2.3). Then for any $G \in L^{2, p}\left(\mathbb{R}^{2}\right)$ with

$$
G=0 \text { on } E \quad \text { and } \quad\|G\|_{L^{2, p}\left(\mathbb{R}^{2}\right)} \leq 1,
$$

we have $|\nabla G(0)| \leq N^{-\epsilon}$.

Lemma 2. For any integer $D \geq 1$ and subset $S \subseteq \gamma$ with $\# S \leq D$, there exists $H \in L^{2, p}\left(\mathbb{R}^{2}\right)$ that satisfies

$$
H=0 \text { on } S, \quad|\nabla H(0)| \geq 1, \quad \text { and } \quad\|H\|_{L^{2, p}\left(\mathbb{R}^{2}\right)} \leq C_{2} D^{5 / p},
$$

where $C_{2}=C_{2}(p)$ depends only on $p$. 
We now prove Theorem 2, presuming the validity of Lemmas 1 and 2 . These lemmas are proven later in the section.

In proving Theorem 2, it suffices to assume that (2.3) holds with $Z$ determined by Lemma 1 .

Let $A \geq 1, D \geq 1$, and let $T: L^{2, p}(E) \rightarrow L^{2, p}\left(\mathbb{R}^{2}\right)$ be an extension operator with norm $A$ and depth $D$. In other terms, for any $f: E \rightarrow \mathbb{R}$,

$$
\begin{aligned}
& T f=f \text { on } E \\
& \|T f\|_{L^{2, p}\left(\mathbb{R}^{2}\right)} \leq A\|f\|_{L^{2, p}(E)}, \text { and } \\
& T f(x)=\sum_{y \in E} \lambda(x, y) f(y) \quad \text { for all } x \in \mathbb{R}^{2},
\end{aligned}
$$

where the coefficients $\lambda(x, y)$ satisfy

$$
\#\{y \in E: \lambda(x, y) \neq 0\} \leq D \text { for all } x \in \mathbb{R}^{2} .
$$

Note that $\lambda(x, y)=\left(T \delta_{y}\right)(x)$, where $\delta_{y}: E \rightarrow \mathbb{R}$ equals 1 at $y$, and equals 0 on $E \backslash\{y\}$. Thus, $\lambda(\cdot, y) \in L^{2, p}\left(\mathbb{R}^{2}\right)$ for each fixed $y \in E$. It follows from the Sobolev theorem that the function $x \mapsto \lambda(x, y)$ belongs to $C^{1}\left(\mathbb{R}^{2}\right)$ for each fixed $y \in E$.

Let

$$
S:=\left\{y \in E: \nabla_{x} \lambda(0, y) \neq 0\right\} .
$$

We claim that $\# S \leq D$. Indeed, suppose for the sake of contradiction that there exist distinct $y_{1}, \ldots, y_{D+1} \in E$ such that $\nabla_{x} \lambda\left(0, y_{k}\right) \neq 0$ for each $k=1, \ldots D+1$. Then, by the implicit function theorem, there exists $x \in \mathbb{R}^{2}$ such that $\lambda\left(x, y_{k}\right) \neq 0$ for each $k=1, \ldots D+1$. This contradicts (2.8), hence proving $\# S \leq D$.

Note that $S \subseteq \gamma($ see $(2.2),(2.9))$. By Lemma 2 there exists $H \in L^{2, p}\left(\mathbb{R}^{2}\right)$ with

$$
H=0 \text { on } S, \quad|\nabla H(0)| \geq 1, \quad \text { and } \quad\|H\|_{L^{2, p}\left(\mathbb{R}^{2}\right)} \leq C_{2} D^{5 / p} \text {. }
$$

Define $F=T\left(\left.H\right|_{E}\right)$. From (2.7),

$$
\nabla F(0)=\sum_{y \in E} \nabla_{x} \lambda(0, y) H(y),
$$

For $y \in S$ the summand vanishes because $H=0$ on $S$, while for $y \in E \backslash S$ the summand vanishes by definition of $S$ (see (2.9)). Therefore, $\nabla F(0)=0$. Finally, (2.5) implies that $F=H$ on $E$, while (2.6) and (2.10) imply that

$$
\|F\|_{L^{2, p}\left(\mathbb{R}^{2}\right)} \leq A\left\|\left.H\right|_{E}\right\|_{L^{2, p}(E)} \leq A\|H\|_{L^{2, p}\left(\mathbb{R}^{2}\right)} \leq C_{2} A D^{5 / p} .
$$

We define $F_{0}:=F-H$. From (2.10) and the above properties of $F$,

$$
F_{0}=0 \text { on } E, \quad\left|\nabla F_{0}(0)\right|=|\nabla H(0)| \geq 1, \quad \text { and } \quad\left\|F_{0}\right\|_{L^{2, p}\left(\mathbb{R}^{2}\right)} \leq\left(C_{2}+1\right) A D^{5 / p} .
$$

Taking $G=F_{0} \cdot\left[\left(C_{2}+1\right) A D^{5 / p}\right]^{-1}$ in Lemma 1, we obtain

$$
N^{-\epsilon} \geq|\nabla G(0)| \geq\left[\left(C_{2}+1\right) A D^{5 / p}\right]^{-1} .
$$

This completes the proof of Theorem 2. In the following subsections we prove Lemmas 1 and 2. 


\subsection{Besov spaces}

The Besov seminorm of a differentiable function $\varphi: \mathbb{R} \rightarrow \mathbb{R}$ is

$$
\|\varphi\|_{\dot{B}_{p}(\mathbb{R})}:=\left(\int_{\mathbb{R}} \int_{\mathbb{R}} \frac{\left|\varphi^{\prime}(s)-\varphi^{\prime}(t)\right|^{p}}{|s-t|^{p}} d s d t\right)^{1 / p} .
$$

The Besov space $\dot{B}_{p}(\mathbb{R})$ consists of functions with finite Besov seminorm.

The Besov and Sobolev spaces are related through the following trace/extension theorem (see [7], [8]).

Theorem 3. Let $\mathcal{R}$ denote the restriction operator $\mathcal{R}(F)=\left.F\right|_{\mathbb{R} \times\{0\}}$, defined for continuous functions $F: \mathbb{R}^{2} \rightarrow \mathbb{R}$.

- The restriction operator $\mathcal{R}: L^{2, p}\left(\mathbb{R}^{2}\right) \rightarrow \dot{B}_{p}(\mathbb{R})$ is bounded. In other terms, $\|\mathcal{R}(G)\|_{\dot{B}_{p}(\mathbb{R})} \leq C_{\mathrm{SB}}\|G\|_{L^{2, p}\left(\mathbb{R}^{2}\right)}$ for every $G \in L^{2, p}\left(\mathbb{R}^{2}\right)$.

- There exists a bounded extension operator $\mathcal{E}: \dot{B}_{p}(\mathbb{R}) \rightarrow L^{2, p}\left(\mathbb{R}^{2}\right)$. In other terms, $\left.\mathcal{E}(g)\right|_{\mathbb{R} \times\{0\}}=g$ and $\|\mathcal{E}(g)\|_{L^{2, p}\left(\mathbb{R}^{2}\right)} \leq C_{\mathrm{SB}}\|g\|_{\dot{B}_{p}(\mathbb{R})}$ for any $g \in \dot{B}_{p}(\mathbb{R})$.

Given $\bar{E}=\left\{s_{1}, \ldots, s_{K}\right\} \subseteq \mathbb{R}$ and $\phi: \bar{E} \rightarrow \mathbb{R}$, where $s_{1}<\cdots<s_{K}$, we denote the Besov trace seminorm of $\phi$ by

$$
\|\phi\|_{\dot{B}_{p}(\bar{E})}:=\inf \left\{\|\varphi\|_{\dot{B}_{p}(\mathbb{R})}: \varphi \in \dot{B}_{p}(\mathbb{R}), \varphi=\phi \text { on } \bar{E}\right\} .
$$

Let $s_{0}:=-\infty$ and $s_{K+1}:=+\infty$. Define

$$
A_{k l}:=\int_{s_{k-1}}^{s_{k}} \int_{s_{l}}^{s_{l+1}} \frac{1}{|s-t|^{p}} d s d t \quad(\text { all } 1 \leq k<l \leq K) .
$$

For $1 \leq k \leq K$, let $n(k) \in\{1, \ldots, K\}$ be such that $s_{n(k)} \in \bar{E}$ is a nearest neighbor of $s_{k}$, and let

$$
m_{k}:=\frac{\phi\left(s_{k}\right)-\phi\left(s_{n(k)}\right)}{s_{k}-s_{n(k)}} .
$$

For $1 \leq k \leq K-1$, let $\Delta_{k}:=\left|s_{k}-s_{k+1}\right|$, and let

$$
M_{k}:=\frac{\left|m_{k}-m_{k+1}\right|}{\Delta_{k}}+\frac{\left|\phi\left(s_{k}\right)+m_{k} \cdot\left(s_{k+1}-s_{k}\right)-\phi\left(s_{k+1}\right)\right|}{\Delta_{k}^{2}} .
$$

The following expression for the Besov trace seminorm can be found in [5] (see Claims 1 and 3 in the proof of Proposition 3.2):

$$
c \cdot\|\phi\|_{\dot{B}_{p}(\bar{E})}^{p} \leq \sum_{k=1}^{K-1} M_{k}^{p} \Delta_{k}^{2}+\sum_{k=1}^{K-1} \sum_{l=k+1}^{K}\left|m_{k}-m_{l}\right|^{p} A_{k l} \leq C \cdot\|\phi\|_{\dot{B}_{p}(\bar{E})}^{p}
$$




\subsection{Proof of Lemma 1}

Recall that $0<\epsilon<1 /(3 p)$. Let $Z \geq 1$ be a parameter, determined before the end of the proof. We assume that (2.3) holds, that is, $N \geq Z$. In this subsection, constants written $C, c$, etc., may depend on $p$ and $\epsilon$, but are independent of other parameters.

For the sake of contradiction, suppose that $G \in L^{2, p}\left(\mathbb{R}^{2}\right)$ satisfies

$$
\begin{aligned}
& G=0 \text { on } E=\left\{\left(2^{-k},\left(2^{-k}\right)^{1+\alpha}\right): k=2, \ldots, N\right\} \cup\{(0,0)\}, \\
& \|G\|_{L^{2, p}\left(\mathbb{R}^{2}\right)} \leq 1 \text { and }|\nabla G(0)| \geq N^{-\epsilon} .
\end{aligned}
$$

Furthermore, by renormalizing $G$ we may assume

$$
N^{-\epsilon} \leq|\nabla G(0)| \leq 1
$$

Let $\delta:=N^{-1 / \alpha}$, and let $\theta \in C_{0}^{\infty}\left(\mathbb{R}^{2}\right)$ satisfy

$$
\begin{aligned}
& \text { (a) } \operatorname{supp}(\theta) \subseteq B(0, \delta), \quad(\text { b) } \theta=1 \text { on } B(0, \delta / 2), \quad \text { and } \\
& \text { (c) }\left|\partial^{\beta} \theta\right| \leq C \delta^{-|\beta|} \text {, whenever }|\beta| \leq 2 .
\end{aligned}
$$

Define $H=\theta G+(1-\theta) J_{0} G$. First we use the Leibniz rule, (2.15.c) and the fact that $H$ is affine on $\mathbb{R}^{2} \backslash B(0, \delta)$ (this follows from (2.15.a)), and then we use the Sobolev theorem (see $(2.1))$ and $\|G\|_{L^{2, p}\left(\mathbb{R}^{2}\right)} \leq 1$, obtaining that

$$
\begin{aligned}
\|H\|_{L^{2, p}\left(\mathbb{R}^{2}\right)} \leq C \cdot\left(\|G\|_{L^{2, p}\left(\mathbb{R}^{2}\right)}\right. & +\delta^{-1}\left\|\nabla G-\nabla J_{0} G\right\|_{L^{p}(B(0, \delta))} \\
& \left.+\delta^{-2}\left\|G-J_{0} G\right\|_{L^{p}(B(0, \delta))}\right) \leq C^{\prime} .
\end{aligned}
$$

From (2.15.b) and $G=0$ on $E$,

$$
H=0 \text { on } E \cap B(0, \delta / 2) .
$$

Note that $\nabla H(0)=\nabla G(0)$, thanks to (2.15.b). Thus, for each $y \in B(0, \delta)$, applying the Sobolev theorem and (2.16) we obtain

$$
|\nabla H(y)-\nabla G(0)|=|\nabla H(y)-\nabla H(0)| \leq C^{\prime}\|H\|_{L^{2, p}\left(\mathbb{R}^{2}\right)}|y|^{\alpha} \leq C^{\prime \prime} \delta^{\alpha}=C^{\prime \prime} N^{-1} .
$$

Note that (2.18) also holds for $y \in \mathbb{R}^{2}$, since $H$ is affine on $\mathbb{R}^{2} \backslash B(0, \delta)$. Since $N$ is sufficiently large (see (2.3)) and $\epsilon<1$, it follows from (2.14) and (2.18) that

$$
c N^{-\epsilon} \leq|\nabla H(y)| \leq C \text { for all } y \in \mathbb{R}^{2} .
$$

Note that $H\left(y_{0}\right)=H\left(y_{1}\right)=0$, where $y_{0}:=(0,0)$ and $y_{1}:=\left(2^{-N}, 2^{-N(1+\alpha)}\right)$, for $N$ sufficiently large. This follows from (2.17), since $y_{1} \in B\left(0, N^{-1 / \alpha} / 2\right)$ when $N$ is sufficiently large. Thus, for $v:=\left(y_{0}-y_{1}\right) /\left|y_{0}-y_{1}\right|$, the mean value theorem implies that $v \cdot \nabla H\left(x^{*}\right)=0$ for some $x^{*} \in B(0, \delta)$ on the line segment joining $y_{0}$ and $y_{1}$. By the Sobolev theorem and (2.16) it follows that

$$
|v \cdot \nabla H| \leq C \delta^{\alpha}=C N^{-1} \quad \text { on } B(0, \delta)
$$


Hence, $\left|\partial_{1} H\right| \leq C^{\prime} N^{-1}$ on $B(0, \delta)$, thanks to the upper bound from (2.19) and the fact $|v-(1,0)| \leq C 2^{-N \alpha}$. Since $H$ is affine on $\mathbb{R}^{2} \backslash B(0, \delta)$, we conclude that

$$
\left|\partial_{1} H(y)\right| \leq C^{\prime} N^{-1} \quad \text { for all } y \in \mathbb{R}^{2} .
$$

Thus, for $N$ sufficiently large, the lower bound in (2.19) and $\epsilon<1$ imply that

$$
\left|\partial_{2} H(y)\right| \geq c^{\prime} N^{-\epsilon} \quad \text { for all } y \in \mathbb{R}^{2} .
$$

We define $\Phi: \mathbb{R}^{2} \rightarrow \mathbb{R}^{2}$ by $\Phi(s, t)=(s, H(s, t))$. The diffeomorphism $\Phi$ maps onto $\mathbb{R}^{2}$ because $\left|\partial_{2} H\right|$ is bounded away from zero (see (2.21)). By (2.19)-(2.21), $\nabla \Phi(x)$ takes the form

$$
\nabla \Phi(x)=\left(\begin{array}{ll}
1 & 0 \\
a & b
\end{array}\right), \quad \text { where } \quad|a| \leq C N^{-1} \quad \text { and } \quad c N^{-\epsilon} \leq|b| \leq C
$$

Thus, $\nabla \Phi(x)$ is invertible for each $x \in \mathbb{R}^{2}$ and

$$
[\nabla \Phi(x)]^{-1}=\left(\begin{array}{cc}
1 & 0 \\
\bar{a} & \bar{b}
\end{array}\right), \text { where } \quad|\bar{a}| \leq \bar{C} N^{\epsilon-1} \quad \text { and } \quad|\bar{b}| \leq \bar{C} N^{\epsilon}
$$

We now define $\Psi=\Phi^{-1}$, and write $\Phi=\left(\Phi_{1}, \Phi_{2}\right)$ and $\Psi=\left(\Psi_{1}, \Psi_{2}\right)$ in coordinates. Differentiating twice the identity $\Psi \circ \Phi=\mathrm{Id}$ shows that

$\nabla \Phi(x) \cdot \nabla^{2} \Psi_{j}(\Phi(x)) \cdot \nabla \Phi(x)=-\sum_{l=1}^{2} \nabla^{2} \Phi_{l}(x) \cdot \partial_{l} \Psi_{j}(\Phi(x)) \quad\left(\right.$ all $\left.x \in \mathbb{R}^{2}, j \in\{1,2\}\right)$.

Now, perform the following operations on the above equation: multiply through twice by $[\nabla \Phi(x)]^{-1}$ (on the left and right), use the identity $\nabla \Psi(\Phi(x))=[\nabla \Phi(x)]^{-1}$, substitute $x=\Phi^{-1}(y)$ on both sides, take $p^{\text {th }}$ powers, sum over $j \in\{1,2\}$, integrate over $y \in \mathbb{R}^{2}$, and perform the change of variable $y=\Phi(x)$ on the right-hand side. Thus, we obtain

$$
\|\Psi\|_{L^{2, p}\left(\mathbb{R}^{2}\right)}^{p} \leq C\|\Phi\|_{L^{2, p}\left(\mathbb{R}^{2}\right)}^{p}\|\operatorname{det}(\nabla \Phi)\|_{L^{\infty}}\left\|(\nabla \Phi)^{-1}\right\|_{L^{\infty}}^{3 p}
$$

Next, insert into $(2.24)$ the bounds $\|\operatorname{det}(\nabla \Phi)\|_{L^{\infty}} \leq C,\left\|(\nabla \Phi)^{-1}\right\|_{L^{\infty}} \leq C N^{\epsilon}$ and $\|\Phi\|_{L^{2, p\left(\mathbb{R}^{2}\right)}}=\|H\|_{L^{2, p}\left(\mathbb{R}^{2}\right)} \leq C^{\prime}$ obtained from (2.22), (2.23) and (2.16). Thus,

$$
\|\Psi\|_{L^{2, p}\left(\mathbb{R}^{2}\right)} \leq C N^{3 \epsilon} .
$$

Define $\varphi=\left.\Psi_{2}\right|_{\mathbb{R} \times\{0\}}$. By (2.25) and Theorem 3,

$$
\|\varphi\|_{\dot{B}_{p}(\mathbb{R})} \leq C_{\mathrm{SB}}\left\|\Psi_{2}\right\|_{L^{2, p}\left(\mathbb{R}^{2}\right)} \leq C^{\prime} N^{3 \epsilon} .
$$

It follows from (2.17) and the definition $\Phi(s, t)=(s, H(s, t))$ that

$$
\Phi(E \cap B(0, \delta / 2)) \subseteq \mathbb{R} \times\{0\}
$$


In coordinates, $\Psi=\Phi^{-1}$ takes the form $\Psi(u, v)=\left(u, \Psi_{2}(u, v)\right)$. Applying $\Psi$ to the previous set containment and using the definition of $\varphi$, we obtain

$$
E \cap B(0, \delta / 2) \subseteq\{(u, \varphi(u)): u \in \mathbb{R}\}
$$

For some integer $K \geq 0$, we write

$$
E \cap B(0, \delta / 2)=\left\{(0,0),\left(2^{-N}, 2^{-N(1+\alpha)}\right), \ldots,\left(2^{K-N}, 2^{(K-N)(1+\alpha)}\right)\right\} .
$$

Thus, $2^{K-N} \geq c \delta$ for some $c>0$. Since $\delta=N^{-1 / \alpha}$, we obtain

$$
K \geq N-C \log (N)
$$

Let $s_{k}:=2^{k-N}$ for $k=1, \ldots, K$, and let $\bar{E}:=\left\{s_{1}, \ldots, s_{K}\right\}$. Define $\phi: \bar{E} \rightarrow \mathbb{R}$ by $\phi\left(2^{k-N}\right)=\left(2^{k-N}\right)^{1+\alpha}$ for $k=1, \ldots, K$.

Next, we apply (2.12) for the $\bar{E}$ and $\phi$ chosen above. The quantity $A_{k l}$ defined in (2.11) satisfies, for all $1 \leq k<l \leq K$,

$$
A_{k l} \geq \int_{2^{k-1-N}}^{2^{k-N}} \int_{2^{l-N}}^{2^{l+1-N}} \frac{1}{|s-t|^{p}} d s d t \geq c \cdot 2^{-(l-N) p} 2^{k-N} 2^{l-N}
$$

Thanks to (2.27), the function $\varphi$ equals $\phi$ on $\bar{E}$. Thus, from (2.12) and (2.29),

$$
\|\varphi\|_{\dot{B}_{p}(\mathbb{R})}^{p} \geq\|\phi\|_{\dot{B}_{p}(\bar{E})}^{p} \geq c \sum_{k=2}^{K-1} \sum_{l=k+1}^{K}\left|m_{k}-m_{l}\right|^{p} \cdot 2^{-(l-N) p} 2^{k-N} 2^{l-N},
$$

where

$$
m_{i}:=\frac{\left(2^{i-N}\right)^{1+\alpha}-\left(2^{i-1-N}\right)^{1+\alpha}}{2^{i-N}-2^{i-1-N}}=\left(2-2^{-\alpha}\right) \cdot 2^{(i-N) \alpha} .
$$

Note that $\left|m_{k}-m_{l}\right| \geq c \cdot 2^{(l-N) \alpha}$ for $2 \leq k<l \leq K$. Inserting this inequality in the above equation, and using $\alpha p=p-2$, we obtain

$$
\|\varphi\|_{\dot{B}_{p}(\mathbb{R})}^{p} \geq c^{\prime} \sum_{k=2}^{K-1} \sum_{l=k+1}^{K} 2^{(l-N)(p-2)} 2^{-(l-N) p} 2^{k-N} 2^{l-N} \geq c^{\prime \prime} \sum_{k=2}^{K-1} 1=c^{\prime \prime} \cdot(K-2) .
$$

Finally, from (2.26) and (2.28), we obtain

$$
c^{\prime \prime} N-C^{\prime \prime} \log (N) \leq\left(C^{\prime}\right)^{p} N^{3 \epsilon p} .
$$

Since $\epsilon<1 /(3 p)$, the above inequality gives a contradiction when $N$ is sufficiently large. Thus, (2.13) cannot hold, completing the proof by contradiction. We now take $Z=Z(\epsilon, p)$ sufficiently large, so that the previous arguments hold for $N \geq Z$. This completes the proof of Lemma 1. 


\subsection{Proof of Lemma 2}

Let $S \subseteq \gamma$ with $\# S \leq D$ be given. For ease of notation, we may assume that $\# S=\bar{D}$. We must construct an $H \in L^{2, p}\left(\mathbb{R}^{2}\right)$ that satisfies (2.4). To start, write

$$
S=\left\{\left(s_{1}, s_{1}^{1+\alpha}\right), \ldots,\left(s_{D}, s_{D}^{1+\alpha}\right)\right\} \quad \text { with } 0 \leq s_{1}<s_{2}<\cdots<s_{D} \leq 1 .
$$

Let $\bar{S}:=\left\{s_{1}, \ldots, s_{D}\right\}$, and define $\phi: \bar{S} \rightarrow \mathbb{R}$ by $\phi\left(s_{k}\right)=\left(s_{k}\right)^{1+\alpha}$ for $k=1, \ldots, D$. Next, we apply $(2.12)$ to this subset $\bar{S}$ and function $\phi$.

We first obtain an estimate on the $A_{k l}$ defined in (2.11):

$$
A_{k l} \leq \int_{-\infty}^{s_{k}} \int_{s_{l}}^{\infty} \frac{1}{|s-t|^{p}} d s d t \leq C \cdot\left|s_{k}-s_{l}\right|^{2-p} \quad(\text { all } 1 \leq k<l \leq D) .
$$

Let $s_{n(k)} \in \bar{S}$ be a nearest neighbor to $s_{k}$, for each $1 \leq k \leq D$, and let

$$
m_{k}:=\frac{\left(s_{k}\right)^{1+\alpha}-\left(s_{n(k)}\right)^{1+\alpha}}{s_{k}-s_{n(k)}} .
$$

From (2.12), (2.30) and $\alpha p=p-2$, there exists $\varphi: \mathbb{R} \rightarrow \mathbb{R}$ such that

$$
\begin{aligned}
& S \subseteq\{(s, \varphi(s)): s \in \mathbb{R}\} \text {, and } \\
& \|\varphi\|_{\dot{B}_{p}(\mathbb{R})}^{p} \leq C \sum_{k=1}^{D-1} \frac{\left|\left(s_{k}\right)^{1+\alpha}+m_{k} \cdot\left(s_{k+1}-s_{k}\right)-\left(s_{k+1}\right)^{1+\alpha}\right|^{p}}{\left|s_{k+1}-s_{k}\right|^{(1+\alpha) p}} \\
& \quad+C \sum_{k=1}^{D-1} \sum_{l=k+1}^{D} \frac{\left|m_{k}-m_{l}\right|^{p}}{\left|s_{k}-s_{l}\right|^{\alpha p}} .
\end{aligned}
$$

By the mean value theorem, each $m_{k}$ takes the form $(1+\alpha) t_{k}^{\alpha}$ for some $t_{k}$ between $s_{k}$ and $s_{n(k)}$. Thus, $\left|m_{k}-m_{l}\right| \leq C\left|t_{k}-t_{l}\right|^{\alpha} \leq C 3^{\alpha}\left|s_{k}-s_{l}\right|^{\alpha}$ for $k \neq l$. (Here, we use the inequalities $\left|t_{k}-s_{k}\right| \leq\left|s_{k}-s_{n(k)}\right| \leq\left|s_{k}-s_{l}\right|$ and $\left|t_{l}-s_{l}\right| \leq\left|s_{l}-s_{n(l)}\right| \leq\left|s_{k}-s_{l}\right|$.) Similarly, $\left|m_{k}-(1+\alpha) s_{k}^{\alpha}\right| \leq C\left|s_{k+1}-s_{k}\right|^{\alpha}$, hence Taylor's theorem provides uniform control on each term from the first sum in (2.32). Therefore,

$$
\|\varphi\|_{\dot{B}_{p}(\mathbb{R})}^{p} \leq C D^{2}
$$

Applying the extension operator $\mathcal{E}$ from Theorem 3 , the function $F=\mathcal{E}(\varphi)$ satisfies $\left.F\right|_{\mathbb{R} \times\{0\}}=\varphi$ and $\|F\|_{L^{2, p}\left(\mathbb{R}^{2}\right)} \leq C_{\mathrm{SB}}\|\varphi\|_{\dot{B}_{p}(\mathbb{R})}$. Thus, from $(2.31)$,

$$
S \subseteq\{(s, F(s, 0)): s \in \mathbb{R}\},
$$

while from (2.33) we obtain

$$
\|F\|_{L^{2, p}\left(\mathbb{R}^{2}\right)} \leq C^{\prime} D^{2 / p} .
$$

We may assume that $\# S \geq 2$, for otherwise Lemma 2 is trivial. Note that $S \subseteq[0,1]^{2}$ lies on a Lipschitz graph. Thus, by (2.34), there exists $s^{*} \in[0,1]$ such that $\left|\partial_{1} F\left(s^{*}, 0\right)\right| \leq C$. By $(2.35)$ and the Sobolev theorem, $\left|\partial_{1} F(0)\right| \leq C^{\prime} D^{2 / p}$. 
Let

$$
M:=\max \left\{\|F\|_{L^{2, p}\left(\mathbb{R}^{2}\right)},\left|\partial_{1} F(0)\right|, 1\right\} .
$$

Without loss of generality, by adding to $F$ some multiple of the coordinate function $(s, t) \mapsto t$, we may assume that $\partial_{2} F(0)=R M$, where $R \geq 1$ shall be determined later. This does not affect statements from the previous two paragraphs. To summarize:

$$
\begin{aligned}
& \left|\partial_{1} F(0)\right| \leq M, \partial_{2} F(0)=R M, \text { and } \\
& \|F\|_{L^{2, p}\left(\mathbb{R}^{2}\right)} \leq M, \text { where } 1 \leq M \leq C^{\prime} D^{2 / p}
\end{aligned}
$$

Pick $\widehat{\theta} \in C_{0}^{\infty}\left(\mathbb{R}^{2}\right)$ that satisfies

$$
\begin{aligned}
& \text { (a) } \operatorname{supp}(\widehat{\theta}) \subseteq[-1,2]^{2} \text {, (b) } \widehat{\theta}=1 \text { on }[-1 / 2,3 / 2]^{2} \text {, and } \\
& \text { (c) }\left|\partial^{\beta} \widehat{\theta}\right| \leq C \text {, whenever }|\beta| \leq 2 \text {. }
\end{aligned}
$$

Define $\widehat{F}:=\theta F+(1-\theta) J_{0} F$.

Mimicking the proof of (2.16) with help from (2.37), (2.38.a), (2.38.c), we obtain

$$
\|\widehat{F}\|_{L^{2, p}\left(\mathbb{R}^{2}\right)} \leq C M .
$$

Mimicking the proof of (2.18) with help from (2.38.a), (2.38.b), (2.39), we obtain

$$
|\nabla \widehat{F}(y)-\nabla F(0)| \leq C^{\prime} M \quad\left(\text { all } y \in \mathbb{R}^{2}\right) .
$$

Now, choose $R$ sufficiently large, determined by $p$, so that the previous inequality and (2.36) imply that

$$
\left|\partial_{1} \widehat{F}(y)\right| \leq C M \quad \text { and } \quad \frac{R M}{2} \leq\left|\partial_{2} \widehat{F}(y)\right| \leq 2 R M \quad\left(\text { all } y \in \mathbb{R}^{2}\right) .
$$

Finally, (2.34),(2.38.b) and $S \subseteq[0,1]^{2}$ imply that

$$
S \subseteq\{(s, \widehat{F}(s, 0)): s \in \mathbb{R}\} .
$$

We define $\Phi: \mathbb{R}^{2} \rightarrow \mathbb{R}^{2}$ by $\Phi(s, t)=(s, \widehat{F}(s, t))$. The diffeomorphism $\Phi$ maps onto $\mathbb{R}^{2}$ because $\left|\partial_{2} \widehat{F}\right|$ is bounded away from zero (see $(2.40)$ ).

We define $\Psi=\Phi^{-1}$. We write $\Phi=\left(\Phi_{1}, \Phi_{2}\right)$ and $\Psi=\left(\Psi_{1}, \Psi_{2}\right)$ in coordinates. As in (2.24), we obtain

$$
\|\Psi\|_{L^{2, p}\left(\mathbb{R}^{2}\right)} \leq C\|\Phi\|_{L^{2, p}\left(\mathbb{R}^{2}\right)} \cdot\|\operatorname{det}(\nabla \Phi)\|_{L^{\infty}}^{1 / p} \cdot\left\|(\nabla \Phi)^{-1}\right\|_{L^{\infty}}^{3} .
$$

It follows from (2.39) and (2.40) that

$\|\Phi\|_{L^{2, p}\left(\mathbb{R}^{2}\right)}=\|\widehat{F}\|_{L^{2, p}\left(\mathbb{R}^{2}\right)} \leq C M,\|\operatorname{det}(\nabla \Phi)\|_{L^{\infty}} \leq 2 R M$ and $\left\|(\nabla \Phi)^{-1}\right\|_{L^{\infty}} \leq C^{\prime}$.

Therefore,

$$
\left\|\Psi_{2}\right\|_{L^{2, p}\left(\mathbb{R}^{2}\right)} \leq\|\Psi\|_{L^{2, p}\left(\mathbb{R}^{2}\right)} \leq C^{\prime \prime} M^{1+1 / p} \leq C^{\prime \prime} M^{3 / 2}
$$


In coordinates, $\Phi(s, t)=(s, \widehat{F}(s, t))$ and $\Psi(u, v)=\left(u, \Psi_{2}(u, v)\right)$, where $\widehat{F}\left(u, \Psi_{2}(u, v)\right)$ $=v$. Applying $\partial_{2}=\partial / \partial v$, setting $u=v=0$, and then using (2.40),

$$
\partial_{2} \Psi_{2}(0)=\left[\partial_{2} \widehat{F}(\Psi(0))\right]^{-1} \geq C M^{-1} .
$$

Finally, (2.41) implies that $S \subseteq \Phi(\mathbb{R} \times\{0\})$. Thus we obtain

$$
\Psi(S) \subseteq \mathbb{R} \times\{0\} .
$$

Let $H=\Psi_{2} / \partial_{2} \Psi_{2}(0)$. The bound $M \leq C \cdot D^{2 / p}$ and (2.42)-(2.44) imply that $H$ satisfies the conclusion of Lemma 2. This completes the proof of Lemma 2.

\section{References}

[1] Fefferman, C.: Extension of $C^{m, \omega}$-smooth functions by linear operators. Rev. Mat. Iberoam. 25 (2009), no. 1, 1-48.

[2] Fefferman, C.: $C^{m}$-extension by linear operators. Ann. of Math. (2) 166 (2007), no. $3,779-835$.

[3] Fefferman, C.: The structure of linear extension operators for $C^{m}$. Rev. Mat. Iberoam. 23 (2007), no. 1, 269-280.

[4] Fefferman, C., Israel, A. And Luli, G.: Sobolev extension by linear operators. J. Amer. Math. Soc. 27 (2014), no. 1, 69-145.

[5] Israel, A.: A bounded linear extension operator for $L^{2, p}\left(\mathbb{R}^{2}\right)$. Ann. of Math (2) 178 (2013), no. 1, 183-230.

[6] Luli, G.: $C^{m, \omega}$ extension by bounded-depth linear operators. Adv. Math. 224 (2010), no. 5, 1927-2021.

[7] Stein, E.M.: The characterization of functions arising as potentials. II. Bull. Amer. Math. Soc. 68 (1962), 577-582.

[8] Triebel, H.: Interpolation theory, function spaces, differential operators. Johann Ambrosius Barth, Heidelberg, 1995.

Received June 10, 2012.

Charles Fefferman: Department of Mathematics, Princeton University, Fine Hall, Washington Road, Princeton, New Jersey 08544, USA.

E-mail: cf@math.princeton.edu

Arie Israel: Courant Institute, New York University, 251 Mercer Street, New York, New York 10012, USA.

E-mail: arie@cims.nyu.edu

Garving K. Luli: Department of Mathematics, University of California at Davis, One Shields Avenue, Davis, CA 95616, USA.

E-mail: kluli@math.ucdavis.edu

The first author is supported by NSF and ONR grants DMS 09-01040 and N00014-08-1-0678. The second author is supported by NSF fellowship DMS 11-03978. The third author is supported by NSF and ONR grants DMS 09-01040 and N00014-08-1-0678. 